Voix et Images

volxetimages

\title{
Cap-aux-Sorciers : un archétype du téléroman québécois
}

\section{Hélène Marchand}

Volume 9, numéro 1, automne 1983

Guy Dufresne

URI : https://id.erudit.org/iderudit/200420ar

DOI : https://doi.org/10.7202/200420ar

Aller au sommaire du numéro

Éditeur(s)

Université du Québec à Montréal

ISSN

0318-9201 (imprimé)

1705-933X (numérique)

Découvrir la revue

Citer cet article

Marchand, H. (1983). Cap-aux-Sorciers : un archétype du téléroman québécois.

Voix et Images, 9(1), 39-40. https://doi.org/10.7202/200420ar d'utilisation que vous pouvez consulter en ligne.

https://apropos.erudit.org/fr/usagers/politique-dutilisation/ 


\title{
Cap-aux-Sorciers: un archétype du téléroman québécois
}

\author{
par Hélène Marchand, Université du Québec à Montréal
}

Visionner le seul épisode qui nous a été accessible du téléroman Cap-auxSorciers de Guy Dufresne, s'est avéré une expérience non seulement agréable, mais fort instructive, et ce, pour deux raisons. La première est toute sentimentale : Cap-aux-Sorciers, diffusé de 1955 à 1958 à l'antenne de RadioCanada, constituait déjà pour nous un des meilleurs souvenirs du début de l'adolescence. Le fait de revoir une de ces émissions, plus de vingt ans plus tard, loin de nous désillusionner sur les bons moments de la télévision naissante, nous a, au contraire, confirmé le dynamisme de cette époque. La deuxième raison tient à des constatations d'ordre technique.

Ce qui frappe de prime abord c'est cette impression de santé et de fougue qui se dégage de Cap-aux-Sorciers, dès le tout début de l'épisode. La caméra se révèle extrêmement mobile, le débit oratoire des comédiens est rapide, syncopé, le dialogue intelligent. Bref, les premières minutes mobilisent déjà toute l'attention du spectateur. Tant et si bien que l'habitué du téléroman canonique, développé en deux décennies, se sent tout à coup bousculé, interpellé, obligé d'écouter.

Nous n'allons pas faire le procès du feuilleton télévisé. Qu'il nous soit permis seulement de nous interroger sur l'indigence des productions qu'on nous sert de façon générale. Sans toutefois être aussi acerbe que les collaborateurs de Liberté, ${ }^{1}$ il faut bien reconnaître que le téléroman semble avoir été conçu pour devenir non pas simplement populaire (ce qui ne l'obligerait en rien à la médiocrité), mais aussi comme un genre de second ordre. À quoi tient cet état de choses? Car enfin, le développement du media télévision ne devait-il pas normalement provoquer l'amélioration du genre téléromanesque plutôt que sa dégradation?

Il nous semble que les conditions artisanales de production qui prévalaient dans les années cinquante constituent l'un des facteurs importants à considérer. L'obligation d'inventer constamment, de s'adapter, tant pour les

1. «Faut voir ça?» Liberté no 141 , vol. 24, no 3, mai-juin 1982. 
auteurs et comédiens que pour les techniciens, stimulait et catalysait les énergies de tous. De plus, les pionniers de la télévision se lançaient dans cette aventure encore imprégnés, on le sait, d'une compétence acquise dans la production radiophonique qui devait arriver à «faire voir» le radioroman. Sans parler de la maîtrise de la représentation que la pratique du théâtre insufflait déjà aux nouveaux artisans du jeune media.

\section{"Le plaisir du texte»}

Nous tenterons, par le biais d'une lecture sémiologique de l'émission visionnée, de mettre en évidence ce jeu harmonieux des uformes de l'expression et du contenu» qui aboutit à une tranche de téléroman admirablement fignolée.

Pour rendre justice à l'œuvre, nous ne nous sommes pas contentés ici de l'émission du 16 janvier 1958, présentée comme spécimen lors de la célébration des vingt-cinq ans de télévision de Radio-Canada. Nous avons tenu compte aussi, dans une mesure moins importante toutefois, de la publication chez Leméac en 1969 du recueil d'épisodes sous-titré Fabienne ${ }^{2}$ et, en partie seulement, du microfilm d'un certain nombre de scénarios déposés à la Bibliothèque nationale. Ces deux éléments du corpus serviront de matériel d'appoint uniquement. Il nous serait difficile, voire impossible, de fonder une analyse sémiologique sur autre chose que la partie de l'œuvre produite en audio-visuel, le téléroman étant conçu avant tout pour être vu.

Il n'est pas superflu de définir nos termes. Nous entendons par lecture sémiologique du téléroman, un travail analytique qui convoque aussi bien les sémiotiques théâtrale et cinématographique que littéraire, et plus précisément narrative, en l'occurrence. ${ }^{3}$ Nous maintenons l'expression «lecture sémiologique» en dépit de l'objection de Pierre Schaeffer, qui nous fournit par ailleurs l'occasion d'expliquer notre démarche :

...si nous avons parlé tout à l'heure de lectures qui varient avec chaque lecteur, nous jugeons bien imprudents ceux qui parlent de «lecture de l'image» ou de "sémiologie du film». C'est que les sons et les images ne mobilisent pas les auditeurs et les spectateurs de la même manière que le langage, où la perception des signes est aussitôt dépassée, oubliée au profit de la signification dont ils sont porteurs. ${ }^{4}$

2. DUFRESNE, Guy, Cap-aux-Sorciers. I Fabienne, Montréal, Leméac, coll. Les Beaux Textes, 1969, $268 \mathrm{p}$.

3. Le mot «sémiologique» est employé ici en tant que terme générique pour signaler l'apport de diverses «sémiologies régionales» à notre lecture. Nous préférons, pour des raisons épistémologiques qu'il serait trop long d'évoquer ici, employer "sémiologie» pour faire référence à l'ensemble des "sémiologies régionales» possibles, et «sémiotique» pour nommer chacune d'entre elles.

4. SCHAEFFER, Pierre dans Helbo, André et al, Sémiologie de la représentation, Bruxelles, Editions Complexe, coll. Creusets, 1975, p. 179. 
Nous convenons avec Schaeffer du fait que chaque lecteur ou spectateur fait sa propre lecture du texte écrit ou présenté, mieux, "représenté», "montré» en audio-visuel. Mais nous considérons que les signes produits dans le téléroman par le travail de la caméra, les décors et costumes, la performance des comédiens, s'additionnent aux signes linguistiques, les dialogues, qui contiennent, quant à eux, la partie la plus «codée» du message. Pour étayer notre opinion et disposer de ces préoccupations théoriques, eu égard aux limites de cet article, nous aurons recours à l'exemple, devenu classique, des «pâtes Panzanni» de Barthes :

...son signifiant est la réunion de la tomate, du poivron et de la teinte tricolore (jaune, vert, rouge) de l'affiche; son signifié est l'Italie, ou plutôt l'italianité; ce signe est dans un rapport de redondance avec le signe connoté du message linguistique (l'assonance italienne du nom Panzanni $) ;(. ..){ }^{5}$

C'est donc au déchiffrement d'un amalgame de systèmes de signes que se livre cette lecture sémiologique.

Ainsi, dans un premier temps nous abordons une lecture du signifiant en examinant les plans et mouvements de la caméra, quelques composantes du jeu des comédiens, etc. Une courte étude narrative constitue ensuite l'amorce d'une analyse du signifié de l'œuvre. Ce programme peut sembler tout de même prétentieux, compte tenu du temps et de l'espace dont nous disposons. C'est pourquoi il ne faut pas chercher dans ces pages un approfondissement de l'usage des disciplines de la kinésique et de la proxémiqué ${ }^{6}$ par exemple, aux fins d'analyses des phénomènes de la représentation dans les créations audio-visuelles; pas plus d'ailleurs qu'une étude exhaustive du récit de Cap-aux-Sorciers. Nous voulons, bien modestement, à l'aide de certaines des méthodes que nous croyons les plus appropriées, ébaucher notre observation des caractéristiques de la dynamique de signification du téléroman de Guy Dufresne.

\section{L'émission du 16 janvier 1958}

Le feuilleton en question raconte la vie du clan du capitaine Aubert, navigateur de la région de Charlevoix. Ce clan se compose d'abord de la famille d'Aubert :

- sa fille, Brigitte, veuve du capitaine Eudore Vigneau qui s'est noyé

- ses petites-filles, Clémence, Fabienne et Anne, «la Nichouette», ainsi que Sylvette, nièce que Brigitte a adoptée,

5. BARTHES, Roland, «Rhétorique de l'image» dans L'Obvie et l'Obtus, Paris, Editions du Seuil, coll. Tel Quel, 1982, p. 27. Cet article est d'abord paru dans Communications en 1964:

6. Pour une description succincte de ces notions, voir Eco, Umberto dans Helbo, André et al., op. cit., pp. 39-40. 
ensuite de:

- Mathilde, une immigrante qui est venue aider «Madame Eudore» après le décès du capitaine Vigneau et s'est intégrée à la famille,

de l'équipage du Saint-Prime :

- Marin, «l'homme de roue» et soupirant officiel de Fabienne

- Firmin, matelot, et enfin de

- Daniel, soupirant officiel d'Anne.

Plusieurs autres personnages, qu'il serait trop long d'énumérer, animent aussi Cap-aux-Sorciers. ${ }^{7}$

L'épisode du 16 janvier 1958 se situe le lendemain des Rois, au moment où la goélette du capitaine Aubert, le Saint-Prime, a subi de graves avaries lors d'un récent naufrage. Aubert doit donc prendre une décision quant à son avenir de navigateur, décision impliquant aussi, évidemment, le sort de sa famille et de son équipage. En plus des membres du clan, qui ne sont pas tous de la distribution de l'émission traitée, apparaissent le personnage de M. Cormier, directeur de la cimenterie (Fabienne est devenue sa secrétaire et l'auberge loge leurs bureaux), le capitaine Bruno LeMarquand rescapé par le Saint-Prime lors du naufrage de son propre bateau ${ }^{8}$ et le capitaine Thivierge, de Québec.

L'épisode est ainsi découpé par la réalisation :

- Séquence $\mathbf{A}$ :

à l'auberge dont le capitaine Aubert est propriétaire

Scène I : Aubert arrive à l'auberge

- Scène II : Aubert rencontre Firmin et Mathilde dans le hall

Scène III : Aubert dans le bureau de Fabienne

Scène IV : Aubert dans le bureau de Cormier

Scène V : . Aubert de nouveau dans le bureau de Fabienne

Scène VI : Aubert rencontre le capitaine Thivierge en quittant l'auberge

Scène VII : - Thivierge rencontre Mathilde et Bruno LeMarquand dans le hall

Scène VIII : Thivierge dans le bureau de Fabienne

Scène IX : Thivierge dans le bureau de Cormier

7. Cette énumération amputée de quelques personnages est empruntée à celle qu'on trouve dans la publication mentionnée en note 2 .

8. Cet épisode est traité dans la publication mentionnée en note 2. 


\section{- Séquence B :}

à la maison jaune, foyer de la famille d'Aubert

Scène I : Fabienne sort de la maison

Scène II : Aubert et Anne dans la chambre des filles

Scène III : Thivierge est reçu au salon par Brigitte, puis Aubert et Anne.

Ce découpage s'appuie donc essentiellement sur les lieux où se déroule l'action et le moment de la journée où elle survient, à savoir, la fin de l'après-midi pour la séquence $A$ et le début de la soirée pour la séquence $B$.

Notre segmentation quant à elle fonctionne au rythme du dévoilement de l'information et pose les principaux jalons de la présente lecture. Elle opère de la façon suivante :

Segment 1 Introduction

Segment 2

Amorce de l'argument de l'épisode

Segment 3

Première digression

Segment 4 Description de l'enjeu

Segment 5

Ajout d'un fait nouveau

Segment 6

Apparition d'un élément potentiellement perturbateur
Séquence $A$, scène I et jusqu'à la dernière partie de la scène II exclusivement :

- Aubert contemple son auberge

- Mathilde et Firmin affirment leur dévouement envers Aubert et l'assurent de la bonne marche de l'auberge

Séquence A, fin de la scène II :

- Aubert rassure Firmin quant à «sa job» sur le Saint-Prime

Séquence A, première partie de la scène III :

- Fabienne s'informe de la santé de Clémence

Séquence A, deuxième partie de la scène III :

- Aubert fait état de la gravité de l'avarie de la goélette; il suppute les prestations d'assurance et fait l'éloge de la carrure du Saint-Prime

Séquence $A$, scènes IV et $V$ :

- Cormier fait une offre alléchante qui pourrait sauver le Saint-Prime

Séquence A, scènes VI, VII, VIII et IX :

- Thivierge serait-il en mesure de profiter de la malchance d'Aubert? 
Segment 7

Deuxième

digression

Segment 8

Epilogue

Segment 9

Dénouement
Séquence B, scène I et première partie de la scène II :

- Aubert s'enquiert de la situation d'Anne et de son fiancé éventuel

Séquence B, deuxième partie de la scène II :

- Anne affirme son statut d'alliée du Saint-Prime

Séquence B, scène III :

- Aubert annonce qu'il renflouera le Saint-Prime

De cette segmentation ressort déjà la présence de deux programmes narratifs dans l'épisode : ceux d'Aubert et de Thivierge. Celui d'Aubert réparti entre les segments $2,4,5,8$ et 9; celui de Thivierge concentré au segment 6 , pour avorter au segment 9 . Deux digressions apparaissent aussi, digressions quant à l'épisode lui-même. Chacune fait allusion à des événements passés et/ou futurs, à des programmes narratifs, l'un ouvert et laissé en suspens (la quête amoureuse d'Anne), l'autre fermé (la quête d'épanouissement personnel de Clémence). Ces digressions raccrochent l'émission au déroulement du téléroman, réaffirment son statut de feuilleton aux divers rebondissements. Le dénouement réinstalle Aubert en tant que sujet/destinateur d'une "quête plus large, celle du succès économique, individuel et collectif, des gens de la région», ${ }^{9}$ programme narratif global d'Aubert et récit enchâssant du téléroman.

\section{La caméra narratrice}

Comme dans le feuilleton télévisé traditionnel, il n'y a pas de véritable narrateur de Cap-aux-Sorciers, si ce n'est la caméra dans son rôle d'intermédiaire entre le plateau où se crée la représentation et le spectateur. Dans le cas qui nous occupe, on peut dire selon Genette, que la caméra narratrice se constitue en observateur "externe» du récit, suivant d'abord Aubert, puis Thivierge dans les péripéties de leur programme narratif respectif, où «le héros agit devant nous sans que nous soyons jamais admis à connaître ses pensées ou sentiments...».10

Le générique du début de l'émission situe d'ailleurs le spectateur à bord d'une goélette qui se dirige vers la rive, sur un village dont on aperçoit distinctement le clocher. Un chœur de quelques hommes constitue la bande sonore de cette intrusion du spectateur au village de Cap-aux-Sorciers :

9. LEGRIS, Renée in Méar, Annie et al., Recherches québécoises sur la télévision, Montréal, Coopératives Albert Saint-Martin, coll. Communications, 1980, p. 36 . A propos d'une émission où Aubert défend les intérêts d'un travailleur de La Malbaie aux prises avec un individu malhonnête. Cet épisode fait partie de la publication mentionnée en note 2.

10. GENETTE, Gérard, Figures III, Paris, Editions du Seuil, coll. Poétique, 1972, p. 207. 
Mais moi je n'en changerai pas

Car la mienne est trop belle

(bis)

au gai

Car la mienne est trop belle... (air connu)

Les voix viriles et cette chanson folklorique soulignent l'atmosphère de terroir et de navigation dans laquelle baigne le téléroman.

\section{Morphologie du personnage d'Aubert}

La scène I de la séquence A s'ouvre sur un plan de demi-ensemble de la partie centrale de la façade d'une maison : murs de pierres des champs, cadrage de la porte en bois, large et percée de carreaux de fenêtre, porte garnie d'un heurtoir. C'est «la maison de pierres» qu'Aubert a quittée à regret avec sa famille pour habiter la «maison jaune» du village, où «Brigitte s'ennuie moins». ${ }^{11}$ Aubert surgit par la gauche, dos à la caméra (plan demi-rapproché). Il se dirige vers la droite (plan américain) et jette un oil sur la plaque à côté de la porte sur laquelle on lit : AUBERGE. Il fait demi-tour, se penche vers la porte (plan américain), actionne le heurtoir. Puis, affichant un air satisfait, comme se moquant de lui-même, il fait de nouveau demi-tour (plan moyen), entre à l'auberge et ferme la porte derrière lui. Après un travelling de la porte à la plaque (gros plan) et de la plaque vers la fenêtre, la caméra traverse celle-ci pour rejoindre Aubert. La chevelure blanche de ce dernier est couverte d'une casquette de marin et il porte un manteau court. Il se tient très droit, les épaules légèrement renversées en arrière. Cette scène qui entame l'introduction, campe le personnage du Capitaine : marin solide, assez âgé, très alerte, fier de ses réalisations, un peu bon enfant.

La scène II de la même séquence $A$ (Aubert rencontre Firmin et Mathilde dans le hall), montre le Capitaine planté bien droit au milieu de la pièce (plan moyen) contemplant l'intérieur de son auberge. Même air satisfait : le hall est constitué d'une salle spacieuse avec cheminée au fond.

Firmin survient par la gauche d'une porte vitrée à deux battants, s'exclamant : «Capitaine!». Manifestement heureux de cette visite, il s'avance vers Aubert. Les deux personnages sont cadrés à gauche en plan américain. Ils échangent des salutations. Une nouvelle exclamation : «Capitaine!» Firmin et Aubert tournent carrément le dos à la caméra (plan américain). À droite de l'écran, en plan moyen, Mathilde descend l'escalier. Elle aussi, heureuse de cette visite, s'approche. Elle lisse ses cheveux tirés en chignon, esquisse un sourire confus. Les trois personnages occupent maintenant tout l'écran en plan américain, Aubert entre Mathilde et Firmin qui respectent une certaine distance entre eux et le Capitaine. Ce dernier leur adresse la parole à tour de rôle, se rapprochant en se penchant légèrement

11. Ce détail se trouve dans la publication mentionnée en note 2 . 
vers eux. Mathilde ne sait que faire de ses mains, visiblement très intimidée. Aubert s'enquiert des activités de chacun, Firmin et Mathilde lui réitèrent leur complet dévouement et cette dernière se retire.

La capitaine se dirige vers le fond de la salle, devant le foyer et redresse en souriant un bateau miniature qui repose sur le manteau de la cheminée. Il tend ses mains au feu. Firmin le suit, le rejoint (les deux personnages en plan américain). Puis, gros plan sur Firmin, qui s'adresse à Aubert. Une conversation s'engage à propos du «bois de corde». Après une pause, Aubert esquissant le geste de quitter les abords de la cheminée, Firmin reprend la parole (il bégaie beaucoup) pour s'inquiéter de son emploi de matelot, au printemps suivant, sur le Saint-Prime (gros plan sur Firmin) : «Ma...ma job su'..su' l' Saint-Prime, j'peux compter d'sus?» Aubert répond (gros plan sur Aubert qui hoche la tête) : «Beau dommage!» Puis, avec une légère moue, le regard se détournant de Firmin pour se projeter au loin : «Hormis qu'y arriverait de quoi... Mais j'vois pas».

La caméra recule (les deux personnages en plan américain), Firmin se retire. Aubert souriant hausse les épaules tendrement, hoche la tête. Il se dirige ensuite vers une porte située au fond de la salle, à gauche de la cheminée. Pour se faire, il se déplace en arc de cerclẹ en jetant de nouveau autour de lui un regard attendri et songeur. Devant la porte (plan italien), il frappe, tourne le bouton et entre.

Cette seconde scène ajoute au palmarès des traits du personnage du Capitaine, celui de patron respecté et admiré de ses employés, qui tiennent à le satisfaire et en qui ils placent leur confiance. Aubert se révèle amical et protecteur.

La troisième scène se passe dans le bureau de Fabienne, exclusivement entre elle et son grand-père. Fabienne est assise derrière sa table de travail, à gauche de l'écran. Aubert apparaît dans la porte (plan moyen). Fabienne se retourne, ravie de reconnaître son grand-père, se lève d'un bond et lui saute au cou. Aubert la prend dans ses bras et l'embrasse délicatement sur les deux joues. Fabienne est volubile et sautillante. Elle insiste pour qu'il retire son manteau qui couvre sa veste de capitaine. Son jeu fait contraste avec le flegme d'Aubert qui ne cache pas cependant un petit air préoccupé. Fabienne s'assoit sur le coin de son pupitre devant Aubert qui occupe un fauteuil (plan moyen). Elle s'informe de Québec (le carnaval commence) et de la santé de sa sœur Clémence (on comprend que Clémence a été forcée de quitter son travail d'infirmière au Pérou pour revenir chez elle, soigner une attaque de malaria).

Puis Fabienne réclame des nouvelles du Saint-Prime. Aubert répond par. une description de la situation financière et des dégâts subis par la goélette (gros plan, puis plan rapproché sur Aubert). Des gestes mesurés de la main illustrent son propos. Il a le front plissé, son regard fixe un point en 
avant, comme l'horizon, et il grimace à l'évocation de l'accident. Ce jeu laisse maintenant percer toute l'inquiétude qui l'habite.

Cette scène donne au personnage du Capitaine ses dimensions de tendresse et de vulnérabilité. Le talon d'Achille d'Aubert c'est sa passion pour la navigation. Privé de la possibilité de naviguer, il ne serait plus lui-même.

La scène IV met en présence le Capitaine et le directeur de la cimenterie, M. Cormier. Ce dernier reçoit Aubert sur le pas de la porte de son bureau, lui serre chaleureusement la main (plan demi-rapproché). Aubert s'assoit devant le pupitre de Cormier qui va se placer debout derrière son fauteuil, près de la fenêtre, et jette un coup d'oeil dehors (plan italien). La conversation s'engage. Cormier parle en contournant sa chaise. Il s'avance, continue de parler, s'immobilise, debout derrière son pupitre. Il s'assoit à son tour. Les deux personnages, maintenant assis, occupent tout l'écran (plan demi-ensemble). Cormier offre à Aubert le commandement d'une barge de fer pour transporter son ciment. La caméra cadre Aubert en plan rapproché. Celui-ci, à la question de Cormier : "Cela vous intéresserait-il?», après une pause, hoche vigoureusement la tête et répond fermement : «Non». Et il continue en entretenant Cormier des avantages qu'offre le transport par, goélette. Il appuie son discours de gestes de la main et en penchant la poitrine vers son interlocuteur. Cormier se lève, passe à côté de son pupitre et s'assoit sur le coin, devant Aubert (la caméra le cadre en plan américain, puis en plan demi-rapproché). Aubert se lève à son tour. (Plan rapproché sur Aubert avec Cormier en second plan) : Cormier offre au Capitaine $\$ 40,000$ d'affaires, par année, pour transporter du ciment par goélette. (Gros plan sur Aubert étonné) : il a un mouvement d'hésitation, son regard se rétrécit, songeur. Il s'informe de la fermeté des intentions de Cormier; ce dernier le rassure sur cette question. L'entrevue est interrompue par la voix de Fabienne qui annonce, par l'appareil de communication à distance, un appel téléphonique d'Ottawa. Cormier fait un mouvement signalant qu'il doit répondre, prend tout de même le temps de serrer la main d'Aubert (plan italien). Le capitaine sort, tandis que Cormier répond en anglais au téléphone.

Cette scène confère au personnage d'Aubert son caractère d'homme d'affaires averti, toujours animé du souci de valoriser la région et ses ressources: L'attitude déférente de Cormier envers son visiteur achève de dépeindre l'estime dans laquelle la communauté tient le capitaine Aubert.

Cette attitude déférente sera reprise dans la scène suivante qui se déroule de nouveau dans le bureau de Fabienne. Quand Aubert revient dans ce bureau, Fabienne est en compagnie de Bruno LeMarquand qui, de toute évidence, lui dicte son rapport d'opérations. LeMarquand et Aubert se saluent. Fabienne s'informe de l'entrevue avec Cormier, Aubert ne révèle rien. Il s'approche de la sortie pour prendre son manteau que LeMarquand s'empresse de décrocher pour aider Aubert à l'enfiler. Celui-çi proteste et 
finit par se laisser faire. Cette scène $V$ clôture et résume la mise en évidence du personnage d'Aubert et laisse en suspens son programme narratif.

Le début de la scène VI où Aubert traverse le hall en reprenant son jeu d'observateur satisfait de l'auberge, fonctionne comme suture entre le segment 5 (l'offre de Cormier) et le segment 6 qui englobe le programme narratif de Thivierge. La caméra devance Aubert dans le hall, passe derrière lui quand il sort et le rejoint dehors en passant par la fenêtre. Cette «sortie» de la caméra, identique à son "entrée» dans l'auberge au début de la séquence, boucle la boucle autour de la construction de l'enjeu de l'épisode du 16 janvier 1958.

\section{Morphologie du personnage de Thivierge}

À peine Aubert a-t-il refermé la porte derrière lui, que survient le capitaine Thivierge vêtu d'un paletot court à carreaux. Les deux hommes se saluent, se serrent la main (plan demi-rapproché). Thivierge en regardant la plaque : "Ç't à vous? Ça marche à vot' goût?» Aubert acquiesce aux deux questions. Thivierge s'informe ensuite du moyen de rencontrer Cormier. Aubert le lui indique et Thivierge annonce sa visite chez Aubert pour après souper. Ils se saluent et Aubert sort du champ, par la gauche. Thivierge se retourne vers la porte, jette un oeil par les carreaux du cadre où apparaît le visage de Mathilde. Elle ouvre et sourit comme il sied à l'hôtesse (plan moyen). Elle recule pour laisser entrer le visiteur (Thivierge est hors champ). Mathilde perd son sourire devant une taquinerie maladroite qu'elle n'apprécie pas et le lui fait savoir.

Ici la caméra «entre» à l'auberge par la porte avant le personnage de Thivierge qui arrive à la suite de Mathilde et du spectateur. Ce procédé instaure une sorte de familiarité avec le spectateur, familiarité dans laquelle Thivierge est inclus après coup, comme à regret.

Ensuite, Thivierge tente de rattraper sa gaucherie envers Mathilde (les deux personnages en plan italien). Celle-ci demande ce qu'elle peut faire pour lui. Thivierge désire souper et coucher, mais Mathilde fait remarquer, en dissimulant mal un certain soulagement, que c'est complet pour la nuit et l'invite à vérifier au registre. Ils se dirigent vers la gauche, au comptoir où Thivierge consulte le registre (plan demi-rapproché). Mathilde recule vers la porte vitrée pour s'éclipser. Thivierge la poursuit, la rattrape, lui effleure le bras et la presse de questions sur le sort d'Aubert (les deux personnages près de la porte vitrée, d'abord en plan demi-rapproché, puis Thivierge en gros plan). Mathilde, très embarrassée, répond qu'elle n'en sait rien. LeMarquand sort du bureau de Fabienne (les trois personnages en plan italien). Mathilde demande à se retirer, mais Thivierge tente de la retenir. LeMarquand s'interpose entre Mathilde et Thivierge (plan américain) en annonçant qu'il y a "promesse» entre Mathilde et lui. Il a posé sa main sur l'épaule de sa fiancée, celle-ci la recouvre de la sienne et le regarde en 
souriant. Thivierge approuve le choix de LeMarquand et félicite le couple. Mathilde se retire par la porte vitrée.

LeMarquand s'avance pour sortir, Thivierge le rejoint et lui demande de souper avec lui. LeMarquand rétorque qu'il ne soupe pas à l'auberge, pas plus qu'il n'y habite. Thivierge remarque en ricanant que, dans les circonstances, cela vaut mieux (Thivierge au second plan, LeMarquand en plan américain). Cette attitude provoque chez LeMarquand une moue d'exaspération; il s'apprête de nouveau à sortir. Thivierge le retient (les deux personnages en plan demi-rapproché) pour s'enquérir du genre d'homme d'affaires qu'est Cormier. LeMarquand répond en brandissant le poing droit, le pouce levé : «Un homme solide».

Cette scène trace un portrait de Thivierge qui s'inscrit presque à l'inverse de celui d'Aubert. Mathilde est mal à l'aise devant Thivierge, non pas parce qu'elle éprouve pour lui respect et admiration, mais bien parce qu'elle se méfie de cet homme aux manières quelque peu grossières. LeMarquand montre un agacement certain et s'empresse d'annoncer ses fiançailles pour libérer définitivement Mathilde des avances plus ou moins sérieuses et malhabiles du Capitaine. L'attitude réceptive de Mathilde envers LeMarquand tranche sur le comportement effarouché que provoque chez elle le capitaine Thivierge et pose ce dernier comme personnage suscitant la «dysphorie».

La scène VIII se déroule dans le bureau de Fabienne qui, assise à son pupitre, fait mine d'être très occupée quand entre Thivierge (elle vient de confier à Aubert qu'elle n'a plus rien à faire). Elle coupe court à la conversation que le visiteur tente d'engager, se contentant d'être la secrétaire courtoise, sans plus, et même avec une certaine sécheresse. Elle annonce Thivierge et lui montre l'accès au bureau de Cormier sans quitter son siège.

Quant à Cormier, il reçoit lui aussi le capitaine Thivierge assis dans son fauteuil, sans cesser de feuilleter des documents. Thivierge se présente, Cormier l'invite à s'asseoir et lui demande ce qu'il peut faire en jetant de nouveau le regard sur ses papiers (plan de demi-ensemble des deux personnages). Thivierge s'inquiète de la méthode de transport du ciment choisie par Cormier. Celui-ci répond d'un air vaguement soupçonneux : «Par eau le plus possible». Thivierge reprend : «Les navigateurs de Cápaux-Sorciers ont dû tomber su' vous comme des taons rapport au charroyage de vot' ciment?» Cormier réplique, malin, comme sachant à quoi s'en tenir sur son interlocuteur : «Non... pas qu' je sache». Thivierge l'avise qu'il songe à se procurer un bateau et qu'il serait prêt à transporter du ciment. Cormier le laisse dire, sourire en coin, légèrement dédaigneux. Quand Thivierge se lève pour partir, Cormier reste assis, informant son visiteur de passer par la porte du fond plutôt que par le bureau de Fabienne vers lequel Thivierge se dirigeait. Le Capitaine sort. La scène se ferme sur 
un fondu au blanc sur le bureau où Cormier, toujours assis à son pupitre (plan demi-rapproché), esquisse un sourire narquois.

Cette attitude partagée par Fabienne et Cormier achève de décrire l'unanimité du clan et de la communauté quant à leur perception du capitaine Thivierge : un individu pas plus désirable qu'il ne faut.

\section{Le langage de la proxémique}

Quiconque a lu des livres comme la Dimension cachée de Hall sait qu'il n'y a aucune modification, si minime soit-elle, de la distance spatiale entre deux individus, qui n'ait une signification différentielle. ${ }^{12}$

Essayons de voir rapidement comment cette «signification différentielle» apparaît à certains moments cruciaux de l'épisode de Cap-aux-Sorciers que nous observons. Nous avons noté plus haut le fait que Mathilde et Firmin respectent l'aire d'Aubert. Nous y avons lu leur révérence pour cet homme âgé, leur employeur et protecteur. De son côté, Aubert ne franchit pas non plus l'espace que ces deux interlocuteurs conservent entre eux et lui. Pour leur signaler son amitié, sa considération, il se penche seulement vers eux, montrant un désir de rapprochement, sans l'imposer. Quant à Fabienne, elle s'empare littéralement de l'espace de son grand-père, elle marque ainsi le degré de son affection. Puis la répartition spatiale entre eux se modifie. Fabienne s'assoit devant son grand-père, sur le coin de son pupitre, se penche vers lui, ils sont dans une certaine proximité, proximité plus amicale que strictement filiale, à ce moment.

Cette même proximité, dans un aménagement identique, se répète dans le bureau de Cormier : celui-ci s'assoit lui aussi sur le coin de son pupitre devant Aubert, au moment où la conversation prend un tour de complicité entre eux. Ils sont ensuite debout, face à face, d'homme à homme, pour poursuivre leur discussion d'affaires. La scène avait débuté par l'accueil de Cormier à la porte et le serrement de main qui réunit deux aires personnelles momentanément; elle s'était poursuivie dans un élargissement de l'espace entre les deux hommes, pour se terminer sur un rapprochement très net.

La rencontre de Thivierge et d'Aubert devant l'auberge se passe à l'extérieur, donc dans un espace aux limites plutôt indéfinies. Leur aire respective se délimite par le diamètre que décrit autour d'eux la distance de l'avant-bras tendu pour serrer la main. Ils sont face a face, sur le même pied, adversaires pour le moment égaux.

Mathilde déploie toutes ses énergies à instaurer le plus d'espace possible entre Thivierge et elle. Les déplacements de la caméra au début de cette scène renchérissent, on l'a vu, sur cette attitude. LeMarquand, quant a lui, saisissant l'embarras de Mathilde, vient occuper l'aire que celle-ci tente de maintenir autour d'elle et que Thivierge ne manquerait pas autrement

12. UMBERTO, Eco dans Helbo, André et al., op. cit., p. 40. 
d'envahir. Mathilde reçoit spontanément LeMarquand dans son aire, confirmant ainsi la réciprocité de son affection. Fabienne et Cormier se laissent tous deux protéger de l'approche physique de Thivierge par le mobilier de leur bureau.

Cette distance constante que les divers personnages maintiennent entre eux et Thivierge monte en épingle les rapprochements spatiaux marquant les scènes centrées sur le capitaine Aubert. Ces signes, produits par la répartition des comédiens dans l'espace, s'agglutinant à ceux des autres composantes du téléroman, raffinent le procès de signification de l'ensemble.

\section{Le regard de la caméra}

Sans faire un relevé exhaustif de tous les plans et mouvements de la caméra, il est possible, en isolant certains d'entre eux, d'amorcer une analyse du fonctionnement du langage visuel.

Concentrons-nous sur le regard que pose la caméra sur les deux personnages centraux de l'épisode du 16 janvier 1958. Dans la séquence A seulement, nous avons noté trois gros plans d'Aubert, pour un seul de Thivierge. Ces gros plans prennent place quand il est question de l'enjeu de l'émission: Aubert cèdera-t-il le Saint-Prime et Thivierge en sera-t-il l'acquéreur?

Séquence $A$, scène II :

Aubert assure «sa job» sur le Saint-Prime à Firmin

Séquence A, scène III :

Aubert commence à expliquer la situation à Fabienne

Séquence $A$, scène IV :

Cormier offre la possibilité de sauver le Saint-Prime

Séquence A, scène VIII :

Thivierge demande à Mathilde si Aubert songe à abandonner la navigation.

Le cadrage du visage de chaque comédien permet d'y lire un certain espoir, de la déception et de l'inquiétude chez Aubert; de la curiosité et une certaine anxiété face à ce qu'il veut apprendre, chez Thivierge. Les gros plans donnent la mesure de la portée du propos du comédien et admettent le spectateur presque jusque dans l'intimité du personnage.

Nous avons aussi remarqué deux plans rapprochés d'Aubert (Séquence A, scènes III et IV, avec Fabienne, puis avec Cormier) alors qu'il est encore explicitement question du Saint-Prime. Ces plans rapprochés jouent sensiblement le même rôle que les gros plans.

De façon générale, Aubert est plus souvent cadré de près que Thivierge et, plus souvent aussi, cadré seul dans l'image. La scène I et le début de la 
scène II de la séquence $\mathrm{A}$, montrent Aubert seul en plans demi-rapproché, américain, italien et moyen, le décrivant abondamment comme on l'a vu plus tôt.

Quant à Thivierge, il apparaît rarement seul dans l'écran, à peine quelques secondes, avons-noụs remarqué, entre le moment où Aubert le quitte devant l'auberge et où on aperçoit le visage de Mathilde par les carreaux du cadrage de la porte (séquence $A$, suture entre les scènes VI et VII). Pendant quelques secondes aussi, il est même totalement absent du champ, comme on l'a souligné plus haut, alors qu'il fait partie de la scène : quand Mathilde lui ouvre la porte de l'auberge et recule pour le laisser entrer.

Ces plans cadrant Thivierge à une certaine distance, toujours mêlé à d'autres personnages, signalent son importance secondaire, en dépit du fait qu'il se pose comme adversaire possible d'Aubert. Ainsi, avant même que la diégèse n'ait relégué Thivierge au second plan, la caméra jette sur lui un regard empreint d'une certaine indifférence.

\section{Les programmes narratifs de l'épisode}

Il pouvait paraître de prime abord étonnant de parler, dès l'étape de la segmentation, de deux programmes narratifs, celui d'Aubert et celui de Thivierge. On aurait pu ne voir au départ dans le récit qu'un seul parcours, celui d'Aubert comme sujet d'une quête centrée sur la possibilité de remettre la goélette en état. Dans ce programme, le clan d'Aubert se constituerait en tant que destinateur de la quête, Cormier, en tant qu'adjuvant et Thivierge, en tant qu'opposant. Or, dès la scène IV de la séquence $A$, Aubert reçoit de Cormier une information qui lui permet d'envisager sérieusement le renflouage du Saint-Prime.

Cette information, modalité du «savoir», conjuguée à celle du «vouloir» d'Aubert (indiqué par sa réponse à Firmin quant à son emploi de matelot et par le non-dit qui se dégage des confidences à Fabienne), procure au Capitaine la modalité du «pouvoir» qui le rend apte à atteindre l'objet de sa quête.

Thivierge survient ensuite, dépourvu à la fois du «savoir» et du «pouvoir» nécessaires à son opposition au projet d'Aubert. Cormier ne lui transmet qu'en partie le «savoir» à propos du mode de transport du ciment. Mais les tentatives répétées de Thivierge auprès des membres du clan d'Aubert pour apprendre ses intentions restent vaines.

Il ne pourra donc pas y avoir de véritable épreuve de force entre le héros et son adversaire, celui-ci ne faisant tout simplement pas le poids. Thivierge ne pourrait être que virtuellement l'opposant d'un programme narratif unique pour décrire le récit de l'émission, car on devine, dès le milieu de l'épisode, que sa démarche est vouée à l'échec. 
C'est pourquoi deux parcours narratifs paralleles, dont les structures actantielles seraient les suivantes, nous semblent mieux schématiser le récit :

Structure actantielle du parcours d'Aubert

$\begin{array}{ll}\text { Destinateur : } & \text { Le clan d'Aubert } \\ \text { Sujet : } & \text { Aubert } \\ \text { Objet : } & \text { Le Saint-Prime } \\ \text { Adjuvant : } & \text { Cormier } \\ \text { Opposants : } & \text { - Le sens des affaires } \\ & \text { - L'âge d'Aubert }\end{array}$

Destinataire : La satisfaction des besoins matériels et psychologiques du clan : gagne-pain de Firmin, fierté et amour de la navigation d'Anne

\section{Structure actantielle du parcours de Thivierge}

Destinateur : Besoin d'améliorer son gagne-pain

Sujet : $\quad$ Thivierge

Objet : $\quad$ Le Saint-Prime

Adjuvant : Le naufrage du Saint-Prime

Opposants : - Le clan d'Aubert

- Le statut d'étranger de Thivierge dans le village de Cap-aux-Sorciers

Destinataire : La satisfaction de Thivierge quant à ses conditions de travail et de rémunération

Dans le parcours d'Aubert, en plus de Firmin et Fabienne qui représentent son clan dans la fonction actantielle de destinateur, il faut intégrer Anne, qui s'inscrira en tant qu'alliée du Saint-Prime à la scène II, de la séquence B. Il faut savoir qu'Anne fait partie de l'équipage de la goélette comme «cook» et qu'elle partage la passion de la navigation de son grandpère. La perspective de voir le bateau tomber en d'autres mains lui répugne, et elle tiendra à accompagner son grand-père lors de l'entretien de la soirée avec Thivierge, histoire de s'assurer qu'Aubert ne cédera pas sa goélette. Anne serait en quelque sorte destinateur/adjuvant, selon son double statut de membre du clan et de soutien et complice de son grand-père.

Les opposants à la quête d'Aubert font piètre figure. Aubert est dépeint chez Cormier comme un homme d'affaires avisé et considéré sur le même pied que le directeur de la cimenterie, lui-même perçu par LeMarquand comme «un homme solide» en affaires. Mais à Anne qui proteste avec émotion à la pensée de voir le capitaine Thivierge devenir le «patron du Saint-Prime», Aubert réplique bien que : «les sentiments... en affaire...", sans montrer pourtant plus de conviction qu'il ne faut. L'âge 
d'Aubert pourrait lui faire songer à la retraite. Ne finit-il pas son monologue sur la carrure du Saint-Prime par ces mots : «bâti fort, ben fort, sur ce côté-là y a pas de goélette su' le fleuve pour l'accoter, bâti pour une vie d'homme», songeant sans doute que cette vie d'homme tire à sa fin pour lui. Et en même temps, on peut avoir l'impression que cette description de la force du bateau rejaillit sur son propriétaire. Il y a bien Brigitte qui fera remarquer avec insistance à la scène III de la séquence $B$, que son père est trop âgé pour songer à construire une nouvelle goélette. Mais Aubert se montre leste et vigoureux, son image n'a rien à voir avec celle du vieillard affaibli et usé.

Le destinateur/destinataire du programme de Thivierge nous est livré quand il confie à Cormier qu'il en a assez de naviguer pour d'autres que luimême et songe à acquérir un bateau. Quant à ses opposants, ils sont de taille : tout le clan d'Aubert, presque la collectivité entière de Cap-auxSorciers pour ainsi dire, qui voit d'un mauvais ceil qu'un Capitaine de Québec vienne chez eux s'emparer d'une de leur meilleure goélette et des contrats de transport du ciment produit dans la communauté.

Quand la séquence A se termine, les protagonistes ont été décrits, leur quête amorcée, le suspense devrait s'être établi. Or, on a vu que dès ce moment, le spectateur est muni de suffisamment d'informations pour deviner l'issue finale. Il faudrait un coup de théâtre, le dévoilement d'une carte mystère dans la manche de Thivierge, par exemple, pour pousser le propriétaire du Saint-Prime à abandonner sa goélette. Cette décision entraînerait d'ailleurs une telle transformation du personnage, qu'elle signifierait la fin du téléroman. Fabienne ne résume-t-elle pas ce trait fondamental d'Aubert quand, à la question de Thivierge : «Le grand-père dans tout ce train-train là y aurait pas perdu le goût de naviguer?», elle répond : «Jamais! C'est dans l' sang!»?

\section{Le dénouement}

La scène I de la séquence $B$, constitue la suture entre les segments 6 et 7 , et introduit le spectateur à la «maison jaune». Mais on peut se demander si cette scène n'a pas une autre fonction. On y voit Fabienne, en tenue de sport, patins en bandoulière, sortir de la maison, détendue et souriante. De toute évidence elle ignore un fait qui la concerne et que le spectateur apprendra avant elle à la scène suivante. ${ }^{13}$

La scène II de la séquence $B$, s'ouvre sur un gros plan d'Anne qui, le visage tourmenté, parle en regardant vers la droite. Elle supplie qu'on la laisse «souffler», tandis que ses sœurs et sa cousine sont à la patinoire, que Marin est à Chicoutimi et Daniel ailleurs. La caméra recule, le cadrage

13. À moins qu'il n'ait regardé les émissions précédentes, et en particulier celle du 9 janvier 1958 où Marin (jusque là soupirant officiel de Fabienne) et Anne se déclarent leur amour mutuel. Source : Microfilm de la Bibliothèque nationale. 
englobe Aubert, debout au second plan, Anne en plan rapproché. Nous sommes dans la chambre des filles. La conversation continue au sujet de la "déclaration» de Marin et du secret qui a trop longtemps entouré cette question selon le grand-père. Il s'approche d'Anne (plan demi-rapproché, derrière la jeune fille en gros plan). Il lui fait remarquer tendrement que sa mère ne sait toujours rien, que c'est "de l'or» cette femme, et qu'elle ne lui pardonnera pas d'avoir fait des cachotteries. «La Nichouette» émue affirme son amour filial et promet que tout sera réglé sous peu. La voix de Brigitte interrompt l'entretien, annonçant le capitaine Thivierge.

Aubert se dirige vers la porte (plan italien). Anne, agacée par cette visite, ne désire pas descendre au salon. Mais Aubert lance : «Y a un œil su' l' Saint-Prime». Anne s'approche d'Aubert : "Lui, patron du SaintPrime!» (plan rapproché des deux personnages, côte à côte). Anne pose la main sur le bras de son grand-père : «...on le verrait passer!» Cette scène entre Anne et Aubert met en évidence leur complicité quand au secret de «la Nichouette» et à leur attachement mutuel au Saint-Prime. Les deux personnages sont cadrés ensemble, Anne se blottit contre son grand-père, elle recherche sa protection et l'assure de son soutien face à Thivierge. Aubert réplique qu'il doit régler cette question lui-même. Elle insiste : «Je descends». Il ne proteste plus : «T'as beau». Cette intimité et cette complicité rappellent celles qui liaient le grand-père et Fabienne lors d'une double quête amoureuse et d'épanouissement personnel de cette dernière, racontée dans un épisode précédent. ${ }^{14}$

La dernière scène montre d'abord Brigitte assise en plan demirapproché, puis Thivierge, en veste de capitaine, assis à sa gauche sur le canapé du salon. Elle ne cache pas son ennui et jette de nombreux regards vers la porte. Le visiteur l'entretient de sa vie de célibataire, bouleversée par sa sœur qui s'est mis en tête de se marier. Il essaie de s'asseoir de plus en plus près de Brigitte qui se tasse désespérément vers la droite pour échapper à ce contact par trop étroit. Elle prend une profonde respiration, lève les yeux au ciel, exaspérée.

Survient enfin Aubert, par la gauche. Il porte aussi sa veste de capitaine. Il salue Thivierge qui se lève et se rassoit. Brigitte montre du soulagement. Aubert est cadré de dos au premier plan, les deux autres font face à la caméra, au second plan. Aubert va s'asseoir sur un banc face au canapé et entreprend de bourrer sa pipe (même cadrage). Anne arrive du fond (plan de demi-ensemble), salue Thivierge, et vient prendre place au premier plan, sur le banc avec Aubert, mais de l'autre côté, faisant face à la caméra. Thivierge fait allusion aux fiançailles prochaines d'Anne qui sursaute avec son grand-père. Elle rétorque vivement pour couper court : «des propos seulement». La caméra fait demi-tour et cadre maintenant

14. Voir à ce propos la publication citée en note 2 et l'analyse de Renée Legris, op. cit., pp. 37- 38. 
Anne de dos et Aubert de face en plan rapproché. Il allume sa pipe et demande au capitaine Thivierge la raison de sa visite. Anne époussette l'épaule d'Aubert. Le visiteur commence à citer tous les on-dit circulant au sujet du Saint-Prime. Aubert plisse les yeux, "La Nichouette» se serre contre lui. Thivierge raconte tous les détails de la situation, les attribuant aux qu'en-dira-t-on. Aubert s'est levé, il fait face à la caméra qui cadre la scène en plan de demi-ensemble, en plongée. Il est surpris, mais rétorque, jouant l'indifférence : «Les marins s'ennuient en hiver». Thivierge poursuit en prétendant qu'Aubert cèderait le Saint-Prime pour $\$ 2000$ comptants puisque ce montant comblerait la différence entre les $\$ 20,000$ offerts par l'assurance et les $\$ 22,000$ qu'espérait le propriétaire de la goélette.

Celui-ci, furieux, se retourne brusquement, s'approche de Thivierge. Anne se lève. Penché sur son interlocuteur, Aubert abandonne son flegme et, ne contenant plus sa colère, réplique presque menaçant, que le SaintPrime n'est pas à vendre. Anne ne se tient plus de joie. Brigitte se lève et offre une tournée de «vin de gadelles». Thivierge, tout à fait décontenancé, n'a pas entendu. Brigitte réitère son offre et Aubert, soudain calmé, presse le visiteur d'accepter. Brigitte sort suivie d'Anne, minaudant.

La caméra cadre ensuite Aubert en gros plan qui annonce à Thivierge qu'il renflouera le Saint-Prime. Ce dernier, déconfit, entre dans le champ (les deux personnages en plan rapproché) et demande à Aubert s'il doublera sa cale en aluminium. De nouveau, gros plan sur Aubert qui répond que cela reste à voir. La scène et l'émission se ferment sur le capitaine Aubert qui regarde au loin, l'air grave.

Ce dénouement, avec sa petite touche inopinée, est traité avec adresse. On se serait attendu à une offre au moins un peu alléchante de la part de Thivierge, ce qui aurait mis Aubert devant un choix difficile. Au contraire, la proposition dérisoire du visiteur, si elle convient au manque de discernement du personnage, confirme l'impression du milieu de l'épisode quant à l'échec certain de sa démarche. Le capitaine Aubert avait probablement presque déjà pris sa décision, mais les propos de Thivierge la précipitent brutalement. La colère, d'abord difficilement contenue d'Aubert, qui éclate enfin, remplace le coup de théâtre avorté, fait rebondir l'action et réanime l'attention au moment de la conclusion.

De plus, le cadrage du salon en plan de demi-ensemble et en plongée, avec Aubert au premier plan, comme haussé par rapport aux autres personnages, le décrivant, dès le milieu de la scène, comme réinstallé dans son rôle de protecteur et défenseur de Cap-aux-Sorciers, ne manque pas de perspicacité. Il faut noter aussi que ce procédé atteste une vigueur dans le travail technique qu'on n'a plus guère le loisir d'admirer dans les téléromans diffusés de nos jours. 


\section{Ce qu'il faudrait aussi étudier dans Cap-aux-Sorciers}

...le destin collectif est assuré par un Destinateur et Sujet, le capitaine Aubert, protecteur du culturel et du social québécois contre l'aliénation. La portée de cette ouvre dramatique dépasse l'anecdote que décrivent beaucoup de feuilletons télévisés traditionnels. ${ }^{15}$

Comme l'exprime avec sagacité Renée Legris, la qualité de Cap-auxSorciers autorise, son inscription en tant qu'archétype du téléroman québécois. Il est loisible de regretter que les concepteurs de feuilletons télévisés ne s'inspirent pas davantage et en plus grand nombre de ce modèle d'intelligence et de finesse.

De plus, la savoureuse langue de Charlevoix et les termes de navigation ajoutent au texte, déjà très riche, ce petit côté exotique qui faisait sans doute rêver de vacances au bord du fleuve, les citadins et ruraux de l'arrière-pays. Fabienne évoque l'atmosphère de l'auberge par ces mots :

Ça fleure la propreté, la soupe aux gourganes, le bois d'érable, les crêpes au sucre, le rôti de lard...»

Et Aubert décrit avec émotion l'avarie du Saint-Prime :

Les glaces ont coupé le bordé, tiens, schoupps, comme au rasoir, à babord, à tribord, su' l' camp, de travers, au ras le fond, au ras le pavois, un dégât terrible. Autant dire un bordé flambant neu (...) Ah! La membrure est «aigrianchée»...

Il fait aussi la promotion de Cap-aux-Sorciers en ces termes :

Vous tombez dans une paroisse de p'tits navigateurs qui construisent eux-mêmes leu' bâtiments de bois. Profitez-en donc. Embauchez donc dix goélettes de 400 tonnes. Y vont vous l' charrier vot' ciment, à meilleur compte.

Si Cap-aux-Sorciers, comme le feuilleton classique, ne déroge pas beaucoup au discours de l'institution, il propose quand même avec subtilité quelques changements sociaux que la «révolution tranquille» ne manquera pas de mettre à la mode quelques années plus tard. L'affirmation du Québécois francophone en est l'exemple le plus évident. On peut, bien sûr, être un peu agacé par les allures paternalistes d'Aubert, et constater que Cap-aux-Sorciers, comme «le téléroman québécois (de cette époque, en général) affirme le pouvoir de l'homme comme Destinateur placé au sommet de la hiérarchie sociale, dont le faire interprétatif est le seul qui peut sanctionner la valeur des quêtes». ${ }^{16}$ Et pourtant, Anne est «cook» à bord du Saint-Prime, ce qui s'avère plutôt original. Dans l'émission du 30 janvier $1958,{ }^{17}$ Aubert défend Anne devant sa mère qui ne peut concevoir qu'elle tienne à naviguer toute sa vie, comme un homme. ${ }^{18}$

15. LEGRIS, Renée, op. cit., p. 38.

16. LEGRIS, Renée, op. cit., p. 45. 
Il y aurait plusieurs autres aspects de Cap-aux-Sorciers à étudier. Le fait de ne pas arriver à épuiser cet ensemble de signes, et ce, même à l'intérieur d'un seul épisode, démontre avec éloquence l'efficacité de l'œuvre. Le téléroman de Guy Dufresne avait légué au genre des lettres de noblesse, malheureusement perdues, du moins en partie, depuis quelques années.

17. Source : Microfilm de la Bibliothèque nationale.

18. Dans le contexte de la navigation, la présence d'une femme dans un équipage était encore, dans les années cinquante, considérée comme pouvant porter malheur. 